\title{
Sub-wavelength plasmonic-enhanced phase-change memory
}

\author{
Emanuele Gemo*a, Santiago García-Cuevas Carrilloa , Joaquin Faneca a , Carlota Ruíz de Galarreta ${ }^{a}$, \\ Hasan Hayat ${ }^{\mathrm{a}, \mathrm{b}}$, Nathan Youngblood ${ }^{\mathrm{c}}$, A. Baldycheva ${ }^{\mathrm{a}}$, Wolfram H.P. Pernice ${ }^{\mathrm{d}}$, Harish Bhaskaran ${ }^{\mathrm{c}}$, \\ C. David Wright ${ }^{\mathrm{a}}$
}

\author{
${ }^{a}$ Department of Engineering, University of Exeter, North Road, Exeter EX4 4QF, UK; \\ ${ }^{\mathrm{b}}$ College of Engineering, Swansea University, Swansea SA1 8EN, UK; \\ ${ }^{c}$ Department of Materials, University of Oxford, Parks Road, Oxford OX1 3PH, UK; \\ ${ }^{\mathrm{d} I n s t i t u t e}$ of Physics, University of Muenster, Heisenbergstrasse 11, 48149 Muenster, Germany
}

\begin{abstract}
The Ge2Sb2Te5 phase-change alloy (GST) is known for its dramatic complex refractive index (and electrical) contrast between its amorphous and crystalline phases. Switching between such phases is also non-volatile and can be achieved on the nanosecond timescale. The combination of GST with the widespread SiN integrated optical waveguide platform led to the proposal of the all-optical integrated phase-change memory, which exploits the interaction of the guided mode evanescent field with a thin layer of GST on the waveguide top surface. The relative simplicity of the architecture allows for its flexible application for data storage, logic gating, arithmetic and neuromorphic computing. Read operation relies on the transmitted signal optical attenuation, due to the GST extinction coefficient. Write/erase operations are performed via the same optical path, with a higher power ad-hoc pulsing scheme, which locally increases the temperature and triggers either the melt-quench process (write) or recrystallization (erase), encoding the information into the GST crystal fraction. Here we investigate the physical mechanisms involved in the write/erase and read processes via computational methods, with the view to explore novel architecture concepts that improve memory speed, energy efficiency and density. We show the achievements of the development of a 3D simulation framework, performing self-consistent calculations for wavepropagation, heat diffusion and phase-transition processes. We illustrate a viable memory optimization route, which adopts sub-wavelength plasmonic dimer nanoantenna structures to harvest the optical energy and maximize light-matter interaction. We calculate both a speed and energy efficiency improvement of around one order of magnitude, with respect to the conventional (non-plasmonic) device architecture.
\end{abstract}

\section{INTRODUCTION}

Phase-change materials (PCMs) exhibit strong variation in their physical properties between amorphous and crystalline phase, and can be switched between such phases quickly (nanosecond or less) and repeatedly (many billions of times), characteristics that are very desirable for many optical and electronic applications ${ }^{1-10}$, in particular for non-volatile memories ${ }^{1-3,10-14}$. Indeed, both optical (e.g. rewritable DVD and Blu-Ray discs) and electrical (e.g. Intel® Optane) commercially available memory devices exploit PCMs. One of the archetypal PCMs is the Ge2Sb2Te5 alloy (GST), which shows a significant variation of both the complex refractive index and the electrical conductivity between phases ${ }^{15-}$ 17. Added to the characteristic transition time on the nanosecond scale and high endurance (i.e. large number of switching cycles), these material properties are of extreme interest for fast and non-volatile photonic applications. Indeed, the combination of GST with the low-loss SiN integrated optical platform led to the proposal of the all-optical integrated phase-change memory ${ }^{18,19}$, which exploits the propagating wave evanescent field interaction with a thin layer of GST deposited on the top surface of the integrated waveguide. In such memory architecture, the phase-state of the GST layer (referred to as optical unit cell) is responsible for the modulation of the waveguide transmission $T$, which is encoded within the optical cell crystal fraction $X$. The concept is illustrated in Fig. 1(a).

The read operation is carried out with a low-power optical probe, whose interaction with the optical cell drives the transmission modulation ${ }^{20}$. Information encoding operations use the same optical path, by delivering relatively high-power pulses. The energy absorbed by the optical cell gives rise to the necessary temperature increase to either trigger GST melting (which is followed by rapid cooling to freeze in the amorphous state), or to induced rapid crystallization (of a previously amorphous cell). More specifically, the amorphization (or write) operation is typically performed by use of a single high-power pulse of short duration (i.e. 1-100 ns) whereas the crystallization (or erase) operation is carried out by 
delivering longer pulses that increase the optical cell temperature above the crystallization temperature but below melting point, inducing both nucleation and crystal growth, depending on local temperature, thermal history, and surface or defect presence. For the erase operation, both pulse trains ${ }^{19}$ and double-step ${ }^{21}$ pulses have confirmed to be effective to achieve a predetermined crystal fraction (and hence the respective transmission modulation). Also, the use of a continuous lowpower read signal is capable to tune the volatility of such memory ${ }^{22}$.

Beyond memory applications, this class of devices can also be applied for novel computing architectures including: arithmetic calculation ${ }^{23}$, neuromorphic processing ${ }^{24}$, and logic gate operations ${ }^{25}$, all of which have been experimentally investigated and demonstrated. Such results pave the way for the photonic implementation of non-von Neumann arithmetic and biologically-inspired computing platforms ${ }^{26}$.

Stemming from the photonic memory concept illustrated above, we here describe a novel concept which takes advantage of the well-known plasmonic field-enhancement effect to further improve the optical interaction with the GST cell ${ }^{27}$. Such a concept combines the aforementioned material-phase dependent optical properties of the GST, with a metallic nanoantenna fabricated on top of the integrated waveguide. The concept is illustrated in Fig. 1(b). The main aim of our design is to maximize light-matter interaction, to reduce the active GST material volume and yet maintain a sufficient contrast between phase-dependent transmission. This directly links with a reduction of energy requirements for write and erase operations, as well as a reduction of the time of the same. Indeed, our numerical findings reveal that such results can be achieved, and in the following sections we illustrate the methodology adopted, the numerical results and our preliminary experimental results, which confirm the predicted plasmonic-enhanced architecture optical behavior.

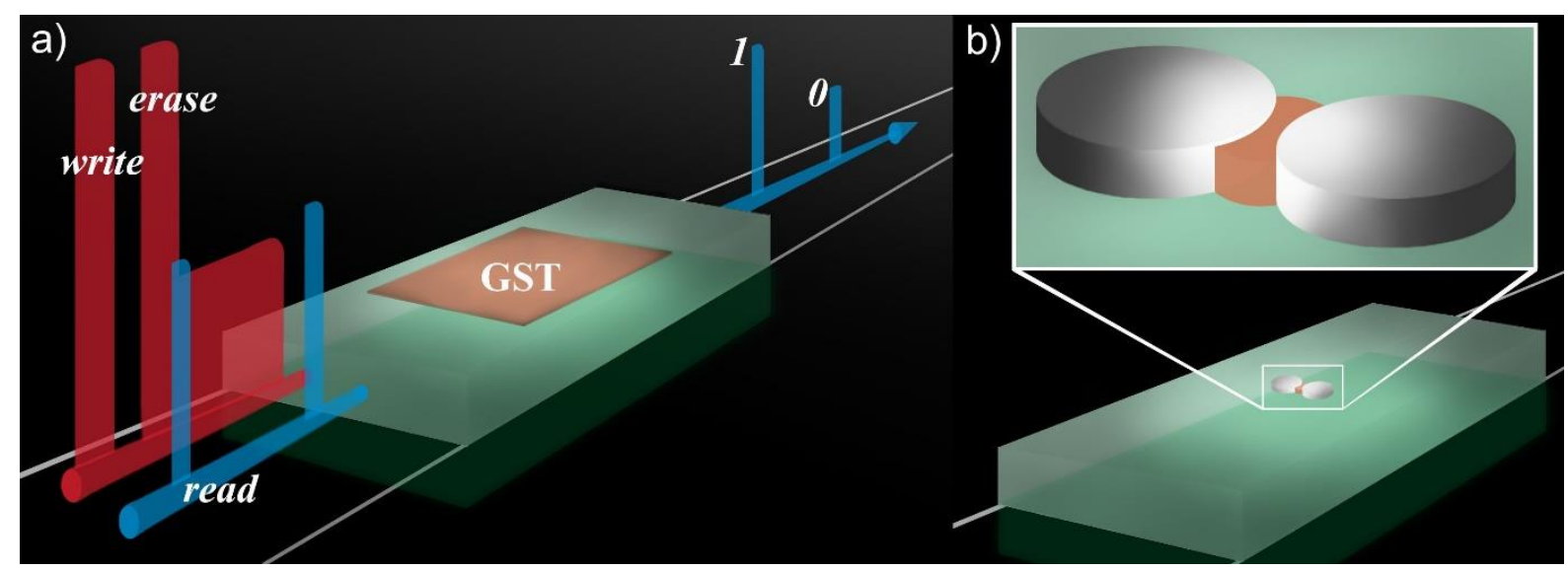

Figure 1. Schematics of the phase-change memory device (green, waveguide; orange, GST; grey, Ag). For scale comparison, the waveguide width is $1300 \mathrm{~nm}$. (a) Conventional architecture and operation principles. Blue beams depict the read operations; red ones represent the write and erase operations. (b) Plasmonic-enhanced architecture.

\section{NUMERICAL METHODS}

To illustrate with sufficient clarity the findings reported in this work, we need to briefly address the numerical research tool and approximations we adopted.

Our numerical investigation relied on the FEM modelling of the conventional photonic memory device, based on the commercial software package COMSOL ${ }^{\circledR}$ along with the MATLAB ${ }^{\circledR}$ environment. Due to the intrinsic 3D nature of the device, the analyzed system can not be described by use of 2D models, which would allow for much faster calculations. Thus, our model relies on a 3D description, which also exploits the planar symmetry (along the propagation axis) to decrease the calculation complexity.

Our model tries to solve the following phenomena: light propagation across the integrated device, heat diffusion arising from the optical energy absorption, and phase transition triggered by the consequent temperature increase. The model geometry consists of a portion of the waveguide, substrate and air cladding, with the GST cell centered within the model. The shape of the investigated model consists of the extrusion of a circular area along the waveguide axis (with the circular areas being perpendicular to the propagation axis), which allows for a reduction of computational requirements while 
minimizing numerical errors. The length of the waveguide portions before and after the device is roughly $1 \mu \mathrm{m}$ to $2 \mu \mathrm{m}$, to allow the correct and artefact-free simulation of the accommodation of the waveguide propagating mode to the deviceinfluenced propagating mode. The width and thickness of the model are roughly $2 \times \lambda$ (with $\lambda$ being the free-space radiation wavelength, here $1550 \mathrm{~nm}$ ), which is sufficient to prevent numerical artefacts and allow for reasonably fast calculations.

For the optical characterization, the waveguide mode input (i.e. the electromagnetic modal profile) is calculated once, as it is invariant during any of the subsequent calculations. The external surfaces are set as $2^{\text {nd }}$ order scattering boundaries, to prevent numerical artefacts, and once more to reduce the computational requirement with respect to the commonly used PML conditions. The symmetry plane boundary condition is set to that of a perfect electric conductor, as the assumed modal profile is TE. The model then solves the wave propagation equation, from which we can determine the following: transmission $T$, reflection $R$, absorption $A$ and diffusion $D$, as well as the space-dependent optical losses.

For the characterization of the temperature variation, the outer surfaces of the model corresponding to the waveguide and the bottom areas are set to fixed ambient temperature, to provide the required model heat sink. The other surfaces (as well as the surfaces belonging to the symmetry plane) are set instead to thermal insulation, which prevents heat from flowing outside the model. The model solves for the heat diffusion equations, taking as a heat source the previously calculated optical volumetric losses (absorbed optical energy).

To simulate the phase transition, which is also responsible for the variation of both the optical and the thermal properties of the GST cell, we implemented the classical nucleation-growth model ${ }^{28}$ in a cellular automata environment, in conjunction with a bespoke melting mechanism. Thus, the phase transition model accounts for three separate phases, whose change is determined by the local temperature (and previous phase-state), the temperature assumed to be nonvariant during each time step. The result of this calculation consists of a space-dependent raster of phase values (crystalamorphous-molten), with a spatial resolution corresponding approximately to the size of the GST fcc crystal lattice ( 0.82 $\mathrm{nm}$ ). The model time-step is determined by use of the Euler method by observation of the main model outputs (crystal fraction, PCM maximum temperature and device transmission). For the characterization of the optical behavior during the read operation, we neglect any temperature increase - phase transition, and assume a stationary state solution (i.e. the solution is not time-dependent during a read). The material optical and thermal parameter adopted for our investigation can be found in Table 1.

Table 1. Adopted optical and thermal material properties ${ }^{27}$.

\begin{tabular}{|l|cccc|}
\hline \multirow{2}{*}{ material } & $\tilde{\mathrm{n}}$ & $\mathrm{C}_{\mathrm{p}}[\mathrm{J} / \mathrm{K}]$ & $\mathrm{K}[\mathrm{W} / \mathrm{mK}]$ & $\delta\left[\mathrm{Kg} / \mathrm{m}^{3}\right]$ \\
\hline $\mathrm{GST}_{\mathrm{cr}}$ & $6.11^{*}+0.83 \mathrm{i}^{*}$ & 210 & 0.58 & 6150 \\
$\mathrm{GST}_{\mathrm{am}}$ & $3.94^{*}+0.045 \mathrm{i}^{*}$ & 210 & 0.2 & 5780 \\
$\mathrm{Si}_{3} \mathrm{~N}_{4}$ & $1.98^{*}$ & $774^{*}$ & $18.4^{*}$ & 2750 \\
$\mathrm{SiO}_{2}$ & 1.44 & $697^{*}$ & $1.37^{*}$ & 2270 \\
$\mathrm{Si}$ & 3.48 & 700 & 157 & 2330 \\
$\mathrm{Ag}$ & $0.145+11.445 \mathrm{i}$ & 235 & 145 & 10820 \\
"Temperature dependent parameters; the stated value refers to room temperature. \\
\hline \multicolumn{5}{l}{} \\
\hline
\end{tabular}

\section{NANOANTENNA OPTICAL BEHAVIOR}

We first focus on the nanoantenna element. In the proposed concept, the nanoantenna couples the propagating mode with the plasmonic resonant mode, whose strength is known to be highly dependent on both size and chemical environment. We find that a relatively simplistic design, consisting of a disc dimer nanoantenna placed symmetrically with respect to the waveguide propagation axis, is highly effective to sustain such behavior. To quantitatively evaluate the resonance strength, we calculate the enhancement factor $(E F)$, determined as the square of the average electric field calculated in the nanoantenna gap, divided by $r$ the (square of the) peak electric field in the unperturbed waveguide. Such resonant strength can be magnified by optimization of four main degrees of freedom: optical properties, nanoantenna radius, thickness and gap sizes.

The material composing the nanoantenna is indeed one of the main factors responsible of such behavior. Our calculation finds that any plasmon-supporting metal is capable to sustain a resonant mode; from literature ${ }^{29}$, we find that silver is 
indeed used in similar photonic nanoantenna designs, spectral ranges and applications, and our simulation study confirms that Ag nanoantennas show stronger resonances than gold or aluminum. From this point onward we will then refer to $\mathrm{Ag}$ nanodiscs. The remaining three degrees of freedom are discs' radius, thickness and size of the gap between them. A simulation study of the effect of these parameters shows how a decreasing gap and thickness allows for stronger enhancement factors, but that the nanodisc radius provides the primary resonance tuning effect. In Fig. 2 we represent the results of our calculation, where along with the $E F$ we evaluated the absolute value of transmission $T$, reflection $R$, absorption $A$ and diffusion $D$. We represent the case for a gap and thickness of $10 \mathrm{~nm}$, and we then report the results for the $E F$ only as a function of the variation of the size of these features.
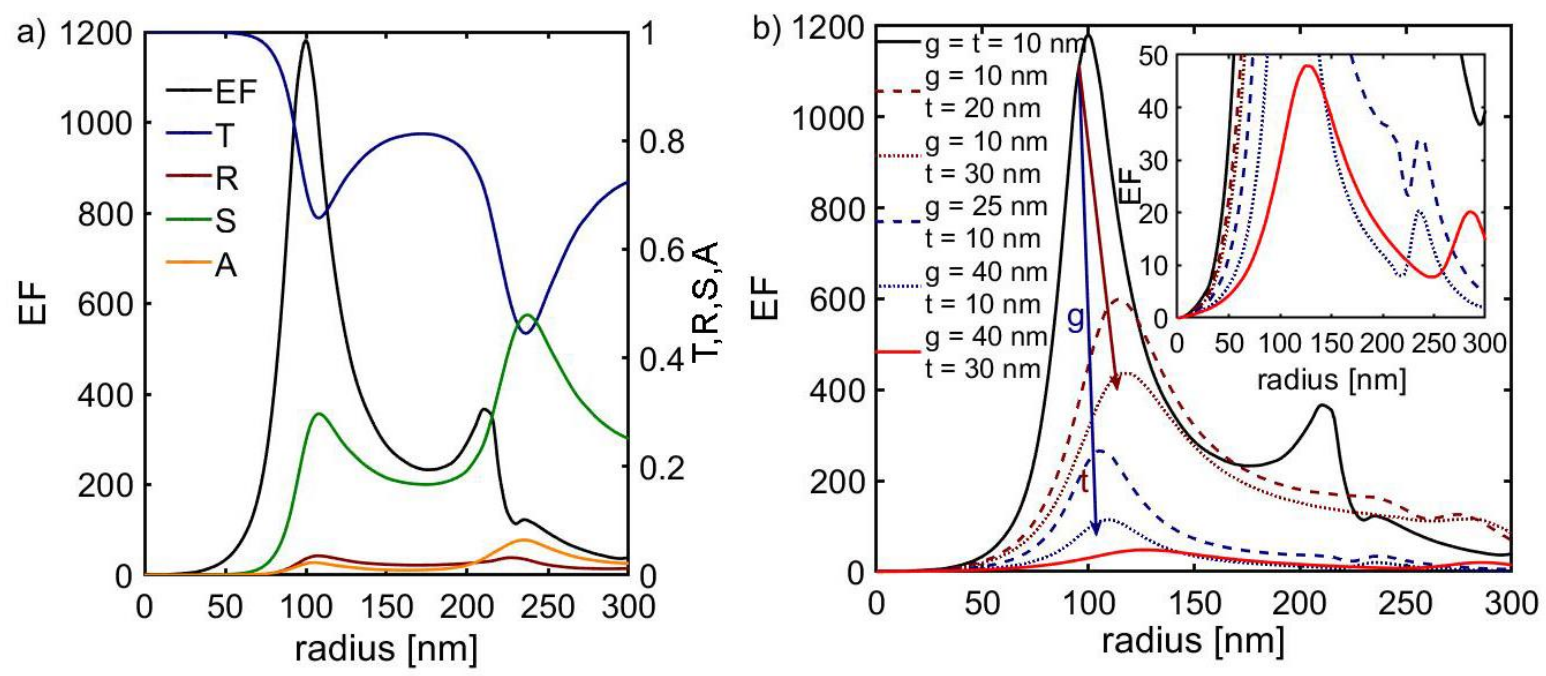

Figure 2. Optical behavior of the devised Ag nanotantenna, fabricated on top of the $\mathrm{Si}_{3} \mathrm{~N}_{4}$ rib waveguide. (a) EF (left axis) and Transmission T, Reflection R, Scattering S, and Absorption A (right axis) for a thickness and gap sizes of $10 \mathrm{~nm}$. The depicted behavior shows two distinct resonances (dipolar, around a value of the radius of $100 \mathrm{~nm}$; quadrupolar, for $210 \mathrm{~nm}$ ), and the consequent modulation of the transmission. The plot also show how the scattering contribution is significantly higher than both absorption and reflection. (b) Comparison of the EF variation as a function of the feature sizes (in legend: $g$ for gap, $t$ for thickness). The inset magnifies the behavior at low values of the EF.

\section{PLASMONIC-ENHANCED MEMORY OPTICAL BEHAVIOR}

After illustrating the nanoantenna behavior, we now turn our attention to what happens when we introduce the PCM (GST) element into the gap region. As mentioned above, the electric field peak is found at the center of the resonant mode field distribution (i.e. within the nanoantenna gap), and our aim is to place the PCM within such narrow cavity. More specifically, in the model we identify the PCM volume as the one contained within a would-be circle of radius $30 \mathrm{~nm}$, centered at the nanoantenna gap.

The PCM confinement within such narrow volume has a multi-faceted justification. First, the aim of this memory design is to enhance the magnitude of the optical field across the PCM cross section, which is indeed achieved by placing its volume entirely within the nanogap, where we demonstrated a theoretical $E F$ up to $10^{3}$ (see Fig. 2(a)). Location of the PCM outside this region has a negligible, if not detrimental, effect on the transmission modulation. More subtly, the variation of the refractive index in the near-field nanoantenna volume has a determinant influence on the resonant strength, which allows the use of the PCM phase-dependent refractive index to tune the EF, and more importantly, the transmission parameter. In Fig. 3 we report the calculation of $E F$ and $T$ as a function of the disc radii, for an optimized configuration for disc thickness and nanogap size. We report two lines for both $E F$ and $T$, color-coded to distinguish the values for the GST amorphous and crystalline phases. As in Fig. 2, we see distinct dipolar and quadripolar resonances, and these show a distinct blue-shift as compared to the 'bare' nanoantenna case. (with the degree of shift greater for crystalline cf. amorphous GST) We also note a decrease of the $E F$ with the introduction of GST in the gap, with the reduction being greater for the crystalline case cf. the amorphous. 

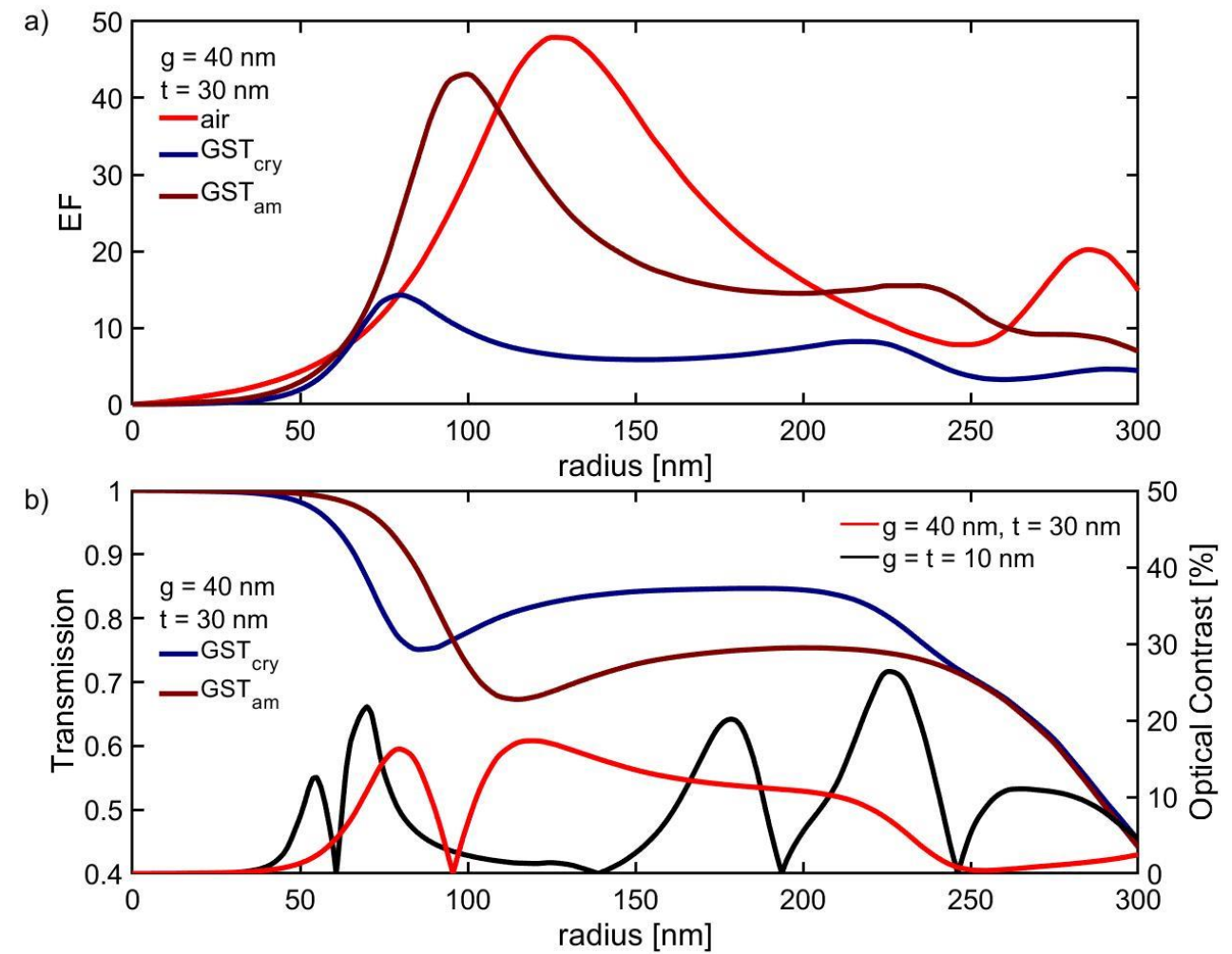

Figure 3. Optical behavior of the plasmonically-enhanced phase-change memory device, for gap and thickness values of 40 $\mathrm{nm}$ and $30 \mathrm{~nm}$ respectively. (a) EF. Red line is reported from Fig. 2(b). The crystalline and amorphous GST configuration show different resonant configurations, thus showing a distinctive blue shift of the resonant peak on the radius axis. (b) Transmission (left axis, navy and wine lines) and optical contrast (right axis, red line). For sake of comparison, the optical contrast for a different configuration is also reported (gap and thickness of $10 \mathrm{~nm}$, black line).

The optical behavior reported above illustrates a memory concept which deviates from the conventional PCM photonic memory architecture, which relies primarily on the optical cell extinction coefficient. In our case, both the extinction coefficient and the refractive index play a crucial role in the determination of the device transmission. The phase-state of the GST cell shifts the optimal nanoantenna resonance peak towards different values of the nanodisc radius, and for such, the optimization of the discs radius requires, among many other parameters, to maximize the change in transmission between the amorphous and the crystal phase configuration. From Fig. 3(b), we determine that a radius value of $75 \mathrm{~nm}$ shows a theoretical transmission of $96 \%$ for the amorphous phase, and $80 \%$ for the crystalline phase, defining a peak optical contrast (defined as $\frac{\left|T_{a m}-T_{c r y \mid}\right|}{T_{\max }}$ ) of $\sim 16 \%$ (for the sake of completeness, the optical contrast is also reported in Fig. 3(b) - black and red lines, right axis).

The values of thickness and nanogap size (of $30 \mathrm{~nm}$ and $40 \mathrm{~nm}$ respectively) reported in Fig. 3 were selected mainly to ease the fabrication process, but we foresee further improvement of the modulation capability with the progress of the fabrication process (see optical contrast data reported in Fig. 3(b), for values of thickness and gap of $10 \mathrm{~nm}$ ). From the fabrication perspective, another factor has been considered. The PCM will be deposited after the nanoantenna fabrication, via an additional lithography step to create the circular window on the resist, followed by sputtering of the PCM helped by use of a calibrated collimator. The PCM would inevitably be deposited also on the top of the discs, but such material would not have any significant effect on the nanoantenna behavior due to the high field localization within the nanoantenna gap. Thus, for sake of clarity, we only consider the PCM material that is in the gap and of the same thickness as the nanodiscs.

\section{FULL 3D TIME-DEPENDENT SIMULATION RESULTS}

By implementation of the full simulation model, we determine that starting from the fully crystalline state a $2 \mathrm{pJ} / 2 \mathrm{~ns}$ write pulse is sufficient to reamorphize most of the PCM volume, shifting the crystal fraction (from 100\%) down to roughly 
$18 \%$, with a corresponding optical contrast value of $12 \%$. The dead time (1/e thermal decay time) is calculated to be 0.6 $\mathrm{ns}$. As for the complete erasure of the written information, we find that a first $2.25 \mathrm{pJ} / 1.5 \mathrm{~ns}$ pulse, followed by a linearly decreasing power pulse of $15 \mathrm{~ns}$ (total energy, $15 \mathrm{pJ}$ ) restores the initial crystal fraction. The complexity of the erase pulse is due to the high temperature and crystal fraction dependence of the absorbed power, which requires the next-to-complete re-amorphization with the first pulse (i.e. melting), followed by a tight control of the PCM temperature in order to start the recrystallization from the external surfaces down to the center of the volume (but without melting the already crystallized areas). The thermal decay time after erasure is calculated to be $0.4 \mathrm{~ns}$. The summary of the write and erase operation can be found in Fig. 4.
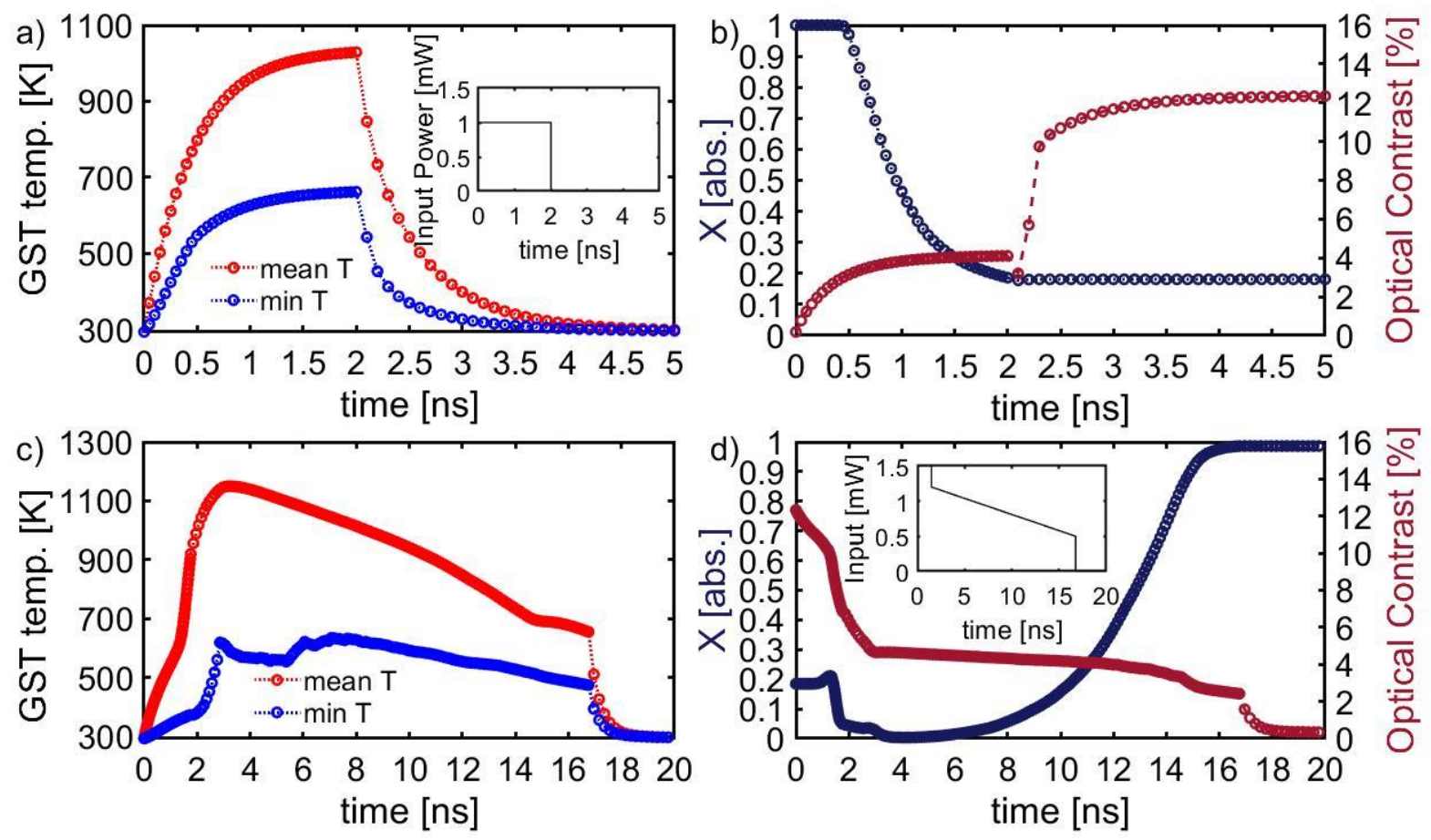

Figure 4. Write and erase operations, as a function of the input power (see insets). Top figures (a,b) report the datasets for the write operation; bottom figures (c,d) report the dataset for the erase operation. (a,c) Temperature profile for the PCM inclusion. (b,d) Cristal fraction (navy, left axis) and optical contrast (burgundy, right axis).

Multi-level memory storage is also achievable, by application of an overwrite operation followed by the controlled application of erase pulse. Termination of the erase pulse before complete recrystallization allows the achievement of a particular desired crystal fraction level. We here report the calculation demonstrating a 4-level memory storage, with optical contrast separation of $\sim 4.2 \%$ between each level (i.e. with contrast of $0 \%, 4.2 \%, 8.3 \%$ and 12.5 , corresponding to the crystal fraction values of $0.18,0.44,0.72$ and 1 respectively). To achieve the same starting crystal fraction for the erase process, we find that the previously described write pulse can be used as an overwrite operation, which notably reduces the crystal fraction to the same approximate level (level $0, \mathrm{X}=18 \%$ ) regardless of the starting point. A summary of the multi-level storage operativity is reported in Fig. 5. 

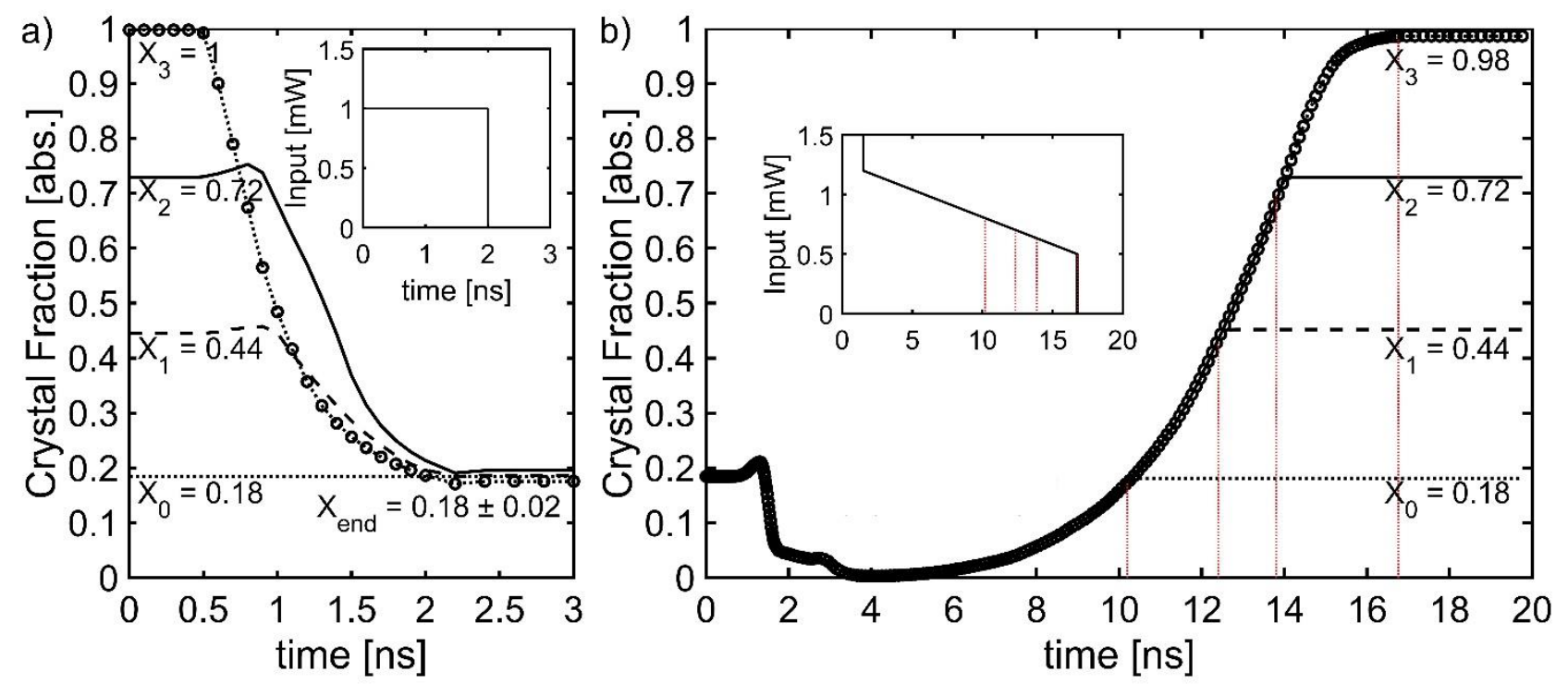

Figure 5. Multi-level operativity summary (crystal fraction values), demonstrating the 2-bit (4 levels) memory storage capability. Inset show the delivered optical power. (a) Overwrite operation, applied from different starting values of the crystal fraction. As visible, after the pulse application each line collapses to the value of $\sim 18 \%$. (b) Erase pulse application effect. Red lines depict the termination of the erase pulse, which allow to reach the previous unique crystal fraction levels.

\section{EXPERIMENTAL RESULTS}

The experimental confirmation of our numerical results is currently being pursued. The photonic memory devices are fabricated using electron beam lithography NBL system on silicon nitride-on-insulator substrates in three exposure steps. The layer stack consists of $300 \mathrm{~nm} \mathrm{Si}{ }_{3} \mathrm{~N}_{4}$ over $2 \mu \mathrm{m} \mathrm{SiO}$, deposited on a silicon carrier wafer. In a first lithography step, windows for gold alignment markers are opened using the PMMA 950K A6 resist spun at $4000 \mathrm{rpm}$ for 60 seconds to have a resist film thickness of $200 \mathrm{~nm}$. The development was carried out in a IPA: MIBK:MEK (15:5:1) mixture for 35 seconds. After depositing $5 \mathrm{~nm}$ of chromium and $50 \mathrm{~nm}$ of gold using the HHV Auto306 thermal evaporator, a lift-off in acetone is carried out and alignment marks for the next lithography steps are patterned.

In a second lithography step, the waveguides are exposed using a TI PRIME adhesion promoter spun at $4000 \mathrm{rpm}$ for 20 seconds and baked at $120^{\circ} \mathrm{C}$ for 120 seconds, and MaN 2403 negative tone resist spun at $3500 \mathrm{rpm}$ for 60 seconds and baked at $90^{\circ} \mathrm{C}$ for 120 seconds. The lift-off is carried out using MF 319 developer for 35 seconds, followed by Reactiveion etching using $\mathrm{SF}_{6}$, to pattern the photonic integrated waveguides on $\mathrm{Si}_{3} \mathrm{~N}_{4}$. The remaining resist is removed in an acetone bath at $80^{\circ} \mathrm{C}$ for 10 minutes, followed by 10 minutes of sonication in the warm acetone at $80 \mathrm{kHz}$. A third and last lithography step consists of opening the windows for the dimer deposition using the same technique used for the markers. The deposition of silver is performed via thermal evaporation and for a thickness of $30 \mathrm{~nm}$. Subsequent resist removal is pursued chemically without mechanical aids (i.e. no sonication applied), to avoid the detachment of the $\mathrm{Ag}$ nanostructures (since no adhesion layer is used). Finally, $5 \mathrm{~nm}$ of $\mathrm{SiO}_{2}$ is deposited to inhibit $\mathrm{Ag}$ oxidation.

The resulting waveguide size, measured by profiler, reports an etching step of $180 \mathrm{~nm}$, for a waveguide thickness of 300 $\mathrm{nm}$ and width of $1300 \mathrm{~nm}$ measured with SEM. All waveguides structures are also connected to input and output grating couplers with a length of $50 \mu \mathrm{m}$, a width of $10 \mu \mathrm{m}$, a period of $1200 \mathrm{~nm}$ and duty cycle of 0.5 . The grating couplers are tapered down to the single-mode waveguide of $1300 \mathrm{~nm}$ width described above.

On the waveguide's top surface, a set of nanoantennas is fabricated (one per waveguide), maintaining a target gap size of $40 \mathrm{~nm}$, and with discs' radii ranging from $50 \mathrm{~nm}$ to $240 \mathrm{~nm}$. The aim is to experimentally verify the numerical prediction of the transmission dependency on the radius size, as illustrated in Fig. 2(a) (blue line). 
Each nanoantenna is characterized by SEM imaging, a typical example being shown in Fig. 6(a). From each image, we extract the nanoantenna average radius and gap size (see the traced lines on top of the nanostructures, Fig 6(a)). The resulting estimation of radii and gap sizes are reported in Figs. 6(b,c). We find that the error of the fabricated features size does not strongly compromise the achievement of the aimed radius, but that achieving the target gap sizes is more challenging (with our current fabrication process).

a)

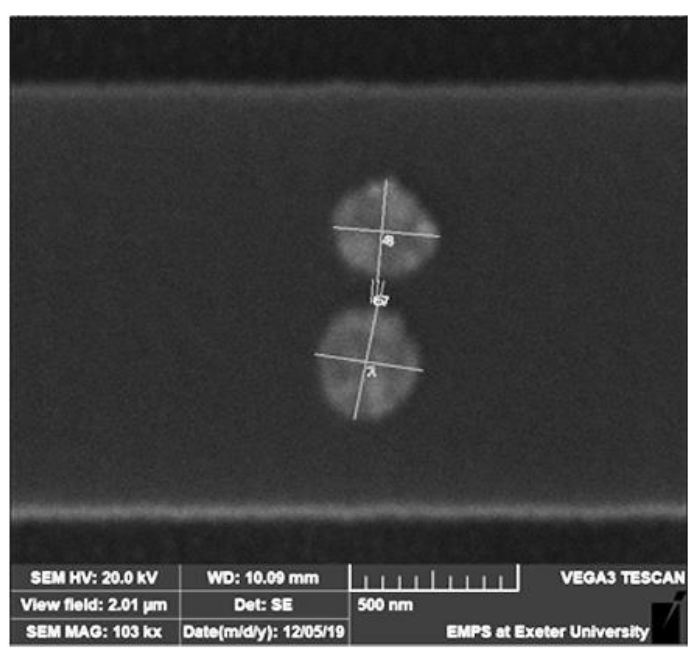

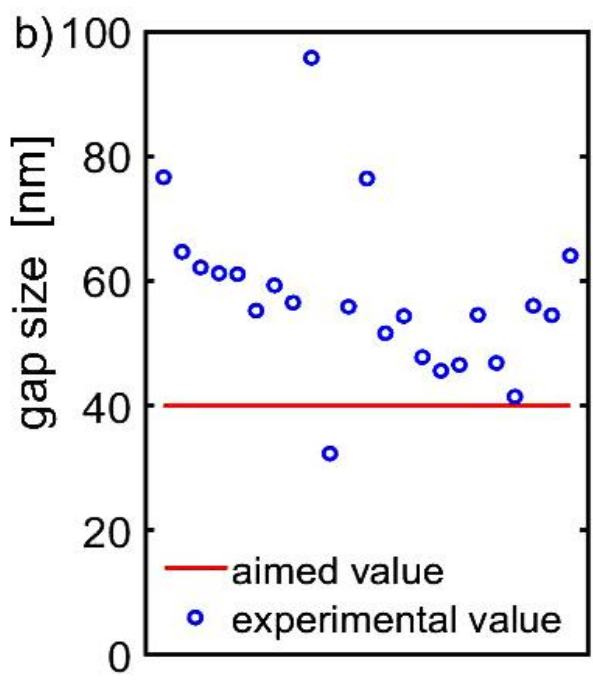

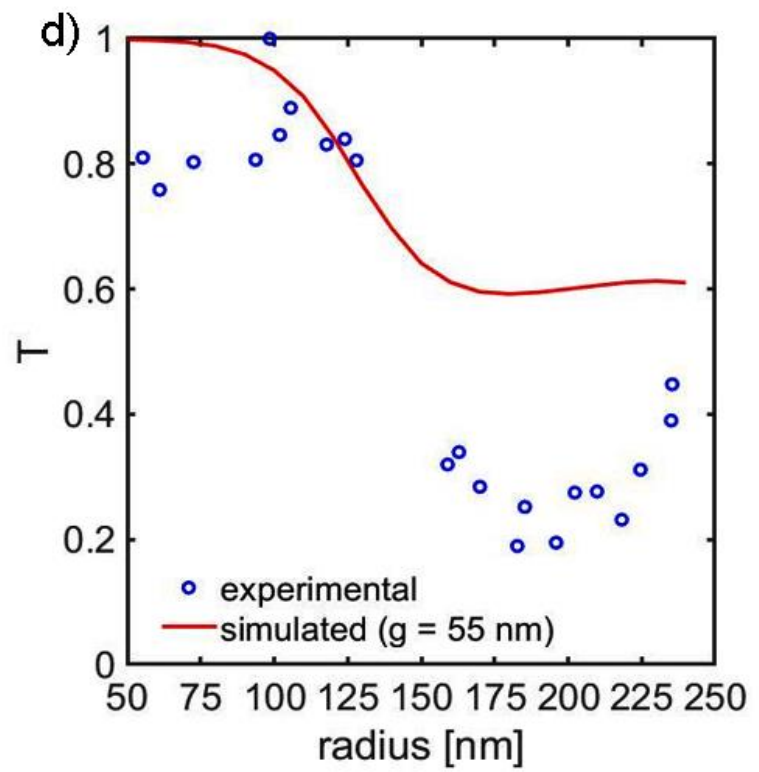

Figure 6. Experimental results of the dimer nanoantennas' fabrication and spectral characterization. (a) SEM image sample of a nanoantenna structure. The aimed radius was $145 \mathrm{~nm}$; we estimate an average radius of $159 \mathrm{~nm}$. The estimated gap size is $76 \mathrm{~nm}$. Estimation traces are visible as white lines on top of the SEM image. (b) Comparison between the aimed gap size (red line) and the experimental values (blue dots). (c) Comparison between the radii aimed values (red line) and experimental values (blue dots). (d) Calculated transmission values (blue dots), compared with the simulation result of a $55 \mathrm{~nm}$ gap nanostructure with varying radius size.

The spectral response (transmission) of each fabricated device was characterized using a fiber-coupled Agilent 8164B tunable laser source, with wavelengths spanning from 1520 to $1610 \mathrm{~nm}$. The light TE polarization is ensured by use of a polarizer controller. Launching of light into the waveguide is by grating couplers, and the angle between the optical fibers 
and the grating couplers is selected to be 14 degrees to ensure maximum coupling at the wavelength of interest (1550 nm). By data prost-processing, we remove the grating coupler and waveguide insertion loss (IL), to calculate the isolated nanoantenna IL contribution. From the nanoantenna IL, we eventually calculate the transmission of the fabricated devices, and compare these with our simulated transmission. For the sake of clarity, we report the trace arising from the simulation of nanostructures with fixed nanogap size of $55 \mathrm{~nm}$ (which is a representative value of the fabrication results). Datasets are shown in Fig. 6(d), from which it can be seen that experimental results and numerical results clearly follow the same trend, where the radius size relates with a non-linear decrease in transmission. In particular, the location of the predicted transmission drop on the radius axis is in good agreement with experimental data (centered at $130 \mathrm{~nm}$ for the simulated dataset, and at $140 \mathrm{~nm}$ for the experimental data). As for the discrepancy between the absolute values of the experimental and simulated transmission, further analysis is indeed required to identify the source of this. A second sample batch is currently being fabricated with the view to improve the features size fabrication reliability and isolate the factors responsible for the found mismatch in absolute values of transmission. It is possible that the source of this inconsistency could be related to a systematic error met during data collection or IL estimation (indeed, normalization of the IL datasets reduces the transmission average error from $\sim 25 \%$ to a much lower value of $4.5 \%$ ).

\section{DISCUSSION AND CONCLUSION}

This work illustrates the progress on the exploration of the viability of a plasmonically-enhanced phase-change integrated photonic memory architecture. This design exploits a relatively simplistic dimer disc Ag nanoatenna, fabricated on the top surface of the integrated waveguide, with the PCM element being placed within the discs' nanogap.

Numerical analysis shows how this concept is capable of harnessing the guided electromagnetic field, enhancing its interaction with the optical cell, and consequently leading to memory operation times (switching times) and energy requirements much smaller than the published data for the conventional architecture. A comparison of the predicted behavior of our devised architecture with a similar conventional device experimental behavior finds a reduction of both write and erase time by 1 order of magnitude, and of the required energy by 1.5 to 2 orders of magnitude. Multi-level storage capability is also predicted; our demonstration is limited to the 2-bit capability, but only due to the arbitrary estimation of a suitable level contrast separation of $\sim 4 \%$.

A downside of the plasmonically-enhanced approach is found in the less straightforward fabrication method, which could eventually be mitigated by advancement of the fabrication techniques and recipes. As for the architecture's intrinsic limitations, we need to also point out a slightly higher insertion loss, due increased scattering contributions.

Our preliminary experimental results show a good match with the identified trends of the nanoantenna's optical behavior as a function of nanoantenna gap and radius sizes, although further analysis is required to justify a seemingly systematic mismatch between numerical and experimental absolute values of the transmission. The next step will be focused on the optimization of the fabrication process, and in the implementation of GST within the nanogap, to confirm the transmission variation as a function of the GST crystallinity, and eventually to verify the possibility to perform reversible optical switching.

\section{FUNDING}

European Union's Horizon 2020 research and innovation program (780848, FunCOMP project); University of Exeter, CDT in Metamaterials (EP/L015331/1); WAFT Collaboration (EP/M015173/1); Chalcogenide Advanced Manufacturing Partnership (ChAMP) (EP/M015130/1); Deutsche Forschungsgemeinschaft (PE1832/5-1).

\section{REFERENCES}

[1] Wuttig, M. and Yamada, N., "Phase-change materials for rewriteable data storage,” Nat. Mater. 6(11), 824-832 (2007).

[2] Wełnic, W. and Wuttig, M., "Reversible switching in phase-change materials," Mater. Today 11(6), 20-27 (2008).

[3] Ielmini, D. and Lacaita, A. L., "Phase change materials in non-volatile storage," Mater. Today 14(12), 600-607 
(2011).

[4] de Galarreta, C. R., Alexeev, A. M., Au, Y. Y., Lopez-Garcia, M., Klemm, M., Cryan, M., Bertolotti, J. and Wright, C. D., "Nonvolatile Reconfigurable Phase-Change Metadevices for Beam Steering in the Near Infrared," Adv. Funct. Mater. 28(10) (2018).

[5] Mohr, D. A., Yoo, D., Chen, C., Li, M. and Oh, S.-H., "Waveguide-integrated mid-infrared plasmonics with high-efficiency coupling for ultracompact surface-enhanced infrared absorption spectroscopy," Opt. Express 26(18), 23540 (2018).

[6] Gholipour, B., Zhang, J., MacDonald, K. F., Hewak, D. W. and Zheludev, N. I., "An all-optical, non-volatile, bidirectional, phase-change meta-switch," Adv. Mater. 25(22), 3050-3054 (2013).

[7] Carrillo, S. G.-C., Nash, G. R., Hayat, H., Cryan, M. J., Klemm, M., Bhaskaran, H. and Wright, C. D., "Design of practicable phase-change metadevices for near-infrared absorber and modulator applications," Opt. Express 24(12), 13563 (2016).

[8] Wang, Q., Rogers, E. T. F., Gholipour, B., Wang, C. M., Teng, J. and Zheludev, N. I., "Optically reconfigurable metasurfaces and photonic devices based on phase change materials," Nat. Photonics 10(1), 60 (2016).

[9] Rudé, M., Mkhitaryan, V., Cetin, A. E., Miller, T. A., Carrilero, A., Wall, S., de Abajo, F. J. G., Altug, H. and Pruneri, V., "Ultrafast and Broadband Tuning of Resonant Optical Nanostructures Using Phase-Change Materials," Adv. Opt. Mater. 4(7), 1060-1066 (2016).

[10] Guo, P., Sarangan, A. and Agha, I., "A Review of Germanium-Antimony-Telluride Phase Change Materials for Non-Volatile Memories and Optical Modulators," Appl. Sci. 9(3), 530 (2019).

[11] Xiong, F., Liao, A. and Pop, E., "Inducing chalcogenide phase change with ultra-narrow carbon nanotube heaters," Appl. Phys. Lett. 95(24), 243103 (2009).

[12] Salinga, M., Kersting, B., Ronneberger, I., Jonnalagadda, V. P., Vu, X. T., Le Gallo, M., Giannopoulos, I., Cojocaru-Mirédin, O., Mazzarello, R. and Sebastian, A., "Monatomic phase change memory," Nat. Mater. 17(8), 681-685 (2018).

[13] Gholipour, B., "Novel Chalcogenide Optoelectronic and Nanophotonic Information Storage and Processing Devices," (Doctoral dissertation, University of Southampton) (2012).

[14] Meena, J., Sze, S., Chand, U. and Tseng, T.-Y., "Overview of emerging nonvolatile memory technologies," Nanoscale Res. Lett. 9(1), 526 (2014).

[15] Park, J.-W., Eom, S. H., Lee, H., Da Silva, J. L. F., Kang, Y.-S., Lee, T.-Y. and Khang, Y. H., “Optical properties of pseudobinary GeTe, Ge2Sb2Te5, GeSb2Te4, GeSb4Te7, and Sb2Te3 from ellipsometry and density functional theory," Phys. Rev. B 80(11), 115209 (2009).

[16] Shportko, K., Kremers, S., Woda, M., Lencer, D., Robertson, J. and Wuttig, M., "Resonant bonding in crystalline phase-change materials," Nat. Mater. 7(8), 653-658 (2008).

[17] Raoux, S., Wełnic, W. and Ielmini, D., "Phase Change Materials and Their Application to Nonvolatile Memories," Chem. Rev. 110(1), 240-267 (2010).

[18] Pernice, W. H. P. and Bhaskaran, H., "Photonic non-volatile memories using phase change materials," Appl. Phys. Lett. 101(17), 171101 (2012).

[19] Ríos, C., Stegmaier, M., Hosseini, P., Wang, D., Scherer, T., Wright, C. D., Bhaskaran, H. and Pernice, W. H. P. P., "Integrated all-photonic non-volatile multi-level memory," Nat. Photonics 9(11), 725-732 (2015).

[20] Rios, C., Stegmaier, M., Cheng, Z., Youngblood, N., Wright, C. D., Pernice, W. H. P. and Bhaskaran, H., "Controlled switching of phase-change materials by evanescent-field coupling in integrated photonics [Invited]," Opt. Mater. Express 8(9), 2455 (2018).

[21] Ríos, C., Youngblood, N., Cheng, Z., Gallo, M. Le, Pernice, W. H. P., Wright, C. D., Sebastian, A. and Bhaskaran, H., "In-memory computing on a photonic platform," Sci. Adv. 5(2), 1-10 (2018).

[22] Youngblood, N., Ríos, C., Gemo, E., Feldmann, J., Cheng, Z., Baldycheva, A., Pernice, W. H. P., Wright, C. D. and Bhaskaran, H., "Tunable Volatility of Ge 2 Sb 2 Te 5 in Integrated Photonics," Adv. Funct. Mater. 29(11), 1807571 (2019).

[23] Feldmann, J., Stegmaier, M., Gruhler, N., Ríos, C., Bhaskaran, H., Wright, C. D. and Pernice, W. H. P., "Calculating with light using a chip-scale all-optical abacus," Nat. Commun. 8(1), 1256 (2017).

[24] Cheng, Z., Ríos, C., Pernice, W. H. P., David Wright, C. and Bhaskaran, H., "On-chip photonic synapse,” Sci. Adv. 3(9) (2017).

[25] Cheng, Z., Ríos, C., Youngblood, N., Wright, C. D., Pernice, W. H. P. and Bhaskaran, H., "Device-Level Photonic Memories and Logic Applications Using Phase-Change Materials," Adv. Mater. 30(32) (2018).

[26] Feldmann, J., Youngblood, N., Wright, C. D., Bhaskaran, H. and Pernice, W. H. P., "All-optical spiking 
neurosynaptic networks with self-learning capabilities," Nature 569(7755), 208-214 (2019).

[27] Gemo, E., Carrillo, S. G.-C., De Galarreta, C. R., Baldycheva, A., Hayat, H., Youngblood, N., Bhaskaran, H., Pernice, W. H. P. and Wright, C. D., "Plasmonically-enhanced all-optical integrated phase-change memory," Opt. Express 27(17), 24724 (2019).

[28] Kalb, J. A., "Crystallization kinetics in antimony and tellurium alloys used for phase change recording," (Doctoral dissertation, University of Aachen) (2006).

[29] Vandenbosch, G. A. E. and Ma, Z., "Upper bounds for the solar energy harvesting efficiency of nano-antennas," Nano Energy 1(3), 494-502 (2012). 